\section{Estudo \\ CoDebate}

em Testão

Plamejamento
Revista Estudo \& Debate, Lajeado, v. 24, n. 3, 2017. ISSN 1983-036X

DOI: http://dx.doi.org/10.22410/issn.1983-036X.v24i3a2017.1276

\title{
ANATOMIA DO CARVÃO DE ESPÉCIES NATIVAS DA REGIÃO DO VALE DO TAQUARI (BIOMA MATA ATLÂNTICA) - BASE DE DADOS PARA ESTUDOS ARQUEOBOTÂNICOS
}

\author{
Mariela Inês Secchi ${ }^{1}$, Leonardo Fluck Mariani ${ }^{2}$, Marjorie Kauffmann ${ }^{1}$, \\ Isa Carla Osterkamp ${ }^{1}$, Soraia Girardi Bauermann ${ }^{3}$, André Jasper ${ }^{1}$
}

\begin{abstract}
Resumo: As análises arqueobotânicas compreendem uma vasta gama de atividades, sendo que dentre elas, o estudo de fragmentos de lenhos carbonizados é uma ferramenta importante para os estudos paleoambientais. Para entender o ambiente pretérito é importante conhecer a vegetação atual para reconstituir, fidedignamente, os paleoambientes. Dessa forma este trabalho visa contribuir para a construção de uma base de dados das características de carvôes vegetais macroscópicos de espécies nativas da região do Vale do Taquari, Rio Grande do Sul. Para isso foram coletados exemplares atuais de 7 famílias, 9 gêneros e 9 espécies, cujos fragmentos foram envoltos em papel alumínio, colocados em cadinhos contendo areia e carbonizados artificialmente em forno mufla, em temperatura de $400^{\circ} \mathrm{C}$ por 40 minutos. Após a carbonização, as amostras foram fotografadas e preparadas para análise em microscopia eletrônica de varredura para análise das estruturas celulares. Com as análises, foi possível perceber uma reduçáo de, em média, 75,57\% de massa das amostras após a carbonização em relação às amostras in natura. Os dados encontrados evidenciaram características anatômicas distintas entre as nove espécies estudadas. Para as duas espécies da família Moraceae, há diferença significativa para todos os parâmetros analisados, enquanto para as espécies de Myrtaceae, os parâmetros diâmetro das pontoaçóes e altura de raios náo foram significativos. Os resultados das medidas avaliadas demonstraram a necessidade de estudos detalhados de anatomia de carvão para a realização de comparaçóes entre material atual e arqueológico para a reconstrução fiel da vegetação pretérita. Esses dados farão parte de um banco de dados antracológico regional, servindo de subsídio para estudos botânicos e arqueobotânicos. Os resultados advindos das análises comparativas futuras que utilizarem a base de dados lançada neste estudo, subsidiarão açóes de gestáo e planejamento ambiental que lidem com a recuperação da cobertura vegetal da regiáo do Vale do Taquari.
\end{abstract}

Palavras-chave: Paleobotânica. Antracologia. Rio Grande do Sul. Coleção de referência.

1 Setor de Botânica e Paleobotânica do Museu de Ciências Naturais da Univates, Programa de Pós-Graduaçáo em Ambiente e Desenvolvimento, Univates. maries@universo.univates.br; marjoriekauffmann@yahoo. com.br. isaosterkamp@hotmail.com; ajasper@univates.br.

2 Acadêmico do curso de Ciências Biológicas - Bacharelado da Universidade do Vale do Taquari - Univates. leo93_mariani@hotmail.com.

3 Laboratório de Palinologia, Universidade Luterana do Brasil. soraia.bauermann@ulbra.br. 


\title{
CHARCOAL ANATOMY OF NATIVE SPECIES FROM THE VALE DO TAQUARI REGION (MATA ATLANTIC BIOME) - DATABASE FOR ARCHAEOBOTANICAL STUDIES
}

\begin{abstract}
The archaeobotanical analyzes comprise a wide range of activities and among them, the study of wood charred fragments is an important tool for paleoenvironmental studies. To understand the past environment is important to know the current vegetation to reconstruct, faithfully, the palaeoenvironments. Thus, the present work aims contribute to the database about the macroscopic charcoal features of native plant species from the Vale do Taquari region, Rio Grande do Sul. For that, specimens of the actual native flora of the area, corresponding to 7 families, 9 genera and 9 species, were collected. The fragments were wrapped in aluminum foil, placed in crucibles containing sand and charred artificially in a muffle furnace at $400^{\circ} \mathrm{C}$ temperature for 40 minutes. After carbonization, the samples were photographed and prepared for analysis by scanning electron microscopy for analysis of cellular structures. Through the analysis, it was possible to notice a reduction of, on average, $75.57 \%$, of mass of the samples after carbonization, in relation to the mass of the samples in natura. The data obtained revealed different characteristics among the nine species. For the Moraceae family, there are significant difference in all the analysed parameters, while for the species of Myrtaceae, the parameters diameter of the pits and height of rays were not significant. The results of the measures evaluated demonstrated the need for detailed charcoal anatomy studies to make comparisons between current and archaeological material for the faithful reconstruction of the past vegetation. These data will be part of an anthracological database that will serve as a base for botanical and archaeobotanical studies. The results from the future comparative analyses using the database presented here, will support environmental management and planning actions that deal with vegetation recovery on the Vale do Taquari region.
\end{abstract}

Keywords: Palaeobotany. Anthracology. Rio Grande do Sul. Reference collection.

\section{Introduçáo e Referencial Teórico}

Durante a pré-história, as sociedades estabeleceram uma economia baseada na utilização de lenha a qual possuía diferentes finalidades, como construção de abrigos; queima para aquecimento e cocção de alimentos; rituais religiosos, dentre outras. Assim, dependendo do processo e/ou finalidade da queima da lenha podem ser encontrados junto ao sedimento em sítios arqueológicos, fragmentos de carvão vegetal macroscópico (charcoal macroscópico).

O carvão é resultado da combustão parcial de elementos vegetais, cujas principais características, segundo Jones e Chaloner (1991) e Scott (2000; 2010), são: coloração negra, brilho sedoso e pintar ao toque (macroscópicas) e paredes celulares homogeneizadas e estruturas celulares bem preservadas (microscópicas).

Devido a sua gênese, Mateus (1996), refere que a carbonização constitui um dos elementos de fossilização mais frequentes em sítios arqueológicos, sendo que sua análise, é eficiente para estudar o relacionamento entre as populaçóes pretéritas e o ambiente, refletindo costumes e técnicas de manejo da vegetação lenhosa de determinado local (MARGUERIE; HUNOT, 2007).

Assim, a Arqueobotânica vem elucidar algumas dúvidas da atualidade, visto que através da utilização de técnicas dessa ciência é possível conhecer os ambientes do passado (vegetação, clima, relação homem versus ambiente) para entender os eventos contemporâneos (TERESO, 2008). 
A Arqueobotânica é o estudo de fragmentos vegetais (e.g. lenhos, folhas, flores, frutos, sementes, pólens) carbonizados ou não, resgatados de sítios arqueológicos com o objetivo de entender a dieta dos povos pretéritos, coleta e cultivo de alimentos e mudanças ambientais (ARCHAEOBOTANY, 2016; BLACK et al., 2006; TERESO, 2007; 2008).

As análises arqueobotânicas compreendem uma vasta gama de atividades (SCHEELYBERT et al.,2003, 2010) sendo que dentre elas, o estudo de grãos de pólen e de fragmentos de lenhos carbonizados são ferramentas importantes para os estudos paleoambientais (HADLER et al., 2012; LEONHARDT, 2007). A correlaçáo destes estudos permite que se façam reconstruçôes do ambiente pretérito, com a possibilidade de explicar fenômenos que ocorrem na atualidade (SCHEEL-YBERT et al., 2010).

Restos de carvão vegetal são comumente encontrados no sedimento em sítios arqueológicos e, até pouco tempo, esses fragmentos eram coletados apenas para fins de datação (SCHEEL-YBERT, 2004 a,b; 2013). A partir da década de 1990, estes fragmentos carbonizados passaram a ser resgatados com maior cuidado, pois refletem a ocorrência de incêndios vegetacionais que tanto podem ser de origem natural quanto antrópica (SCHEEL et al., 1996; SCHEEL-YBERT, 2000; SCOTT, 2000; 2010).

A análise do carvão vegetal permite auxiliar no conhecimento da vegetação e do clima pretérito da área onde o sítio arqueológico estava inserido, e entender de que forma as populaçóes pré-coloniais se relacionavam com o ambiente do entorno (SCOTT; DAMBLON, 2010). A análise de carvão vegetal macroscópico ou antracologia permite, também, que se façam inferências paleoetnobotânicas, pois os vestígios arqueobotânicos encontrados no sítio arqueológico podem ser interpretados como produto da relação entre as populaçóes pré-coloniais com o meio onde viviam (SCHEEL-YBERT et al.,2010).

A interpretação dos dados paleoambientais e paleoclimáticos do Quaternário é feita por comparação com sistemas atuais, por isso, os bancos de dados de lenhos carbonizados são ferramentas importantes para o conhecimento da vegetação atual, para que seja possível uma reconstituição fiel dos ambientes pretéritos, buscando auxiliar na resposta de questóes paleoetnobotânicas (SCHEEL-YBERT et al., 2006).

Trabalhos realizados por Schmidt (2010), Beuren et al. (2012) e Secchi (2012) tentaram identificar os fragmentos carbonizados encontrados em sítios arqueológicos de origem Guarani no Vale do Taquari, RS, mas devido à falta de coleção de referência, essa identificação tornou-se difícil.

Além disso, estudos realizados por autores como Afonso et al. (2015), Gonçalves et al. (2012), Kim e Hanna (2006), Muñiz et al. (2013), Nisgoski et al. (2014), Prior e Alvin (1983); Prior e Gasson (1993), Scheel-Ybert et al. (2010) demonstraram que a estrutura anatômica da madeira se preserva após a carbonização, sendo possível a identificação das espécies arbóreas pretéritas através de comparação com carvão da flora atual. As análises de carvão de lenhos contemporâneos e a elaboraçáo de um banco de dados antracológico visa subsidiar estudos arqueobotânicos através da comparação dos fragmentos carbonizados encontrados em contexto arqueológico com os atuais.

Assim, o presente estudo tem por objetivo iniciar a construção de uma base de dados acerca das características de carvóes vegetais macroscópicos produzidos artificialmente a 
partir de lenhos contemporâneos de espécies nativas da região do Vale do Taquari, Rio Grande do Sul. Estas informaçóes constituirão referência para inferências paleoambientais e paleoetnológicas relacionadas à área de estudo, permitindo a definição de processos de planejamento e gestão ambiental regional.

\section{Material e Métodos}

Foram coletadas amostras de ramos lenhosos com $10 \mathrm{~cm}$ de comprimento, e diâmetro superior a $1,0 \mathrm{~cm}$, de diferentes espécimes da flora nativa (QUADRO 01), na região do Vale do Taquari, em um fragmento de Mata Atlântica na localidade de Costão, Estrela, Rio Grande do Sul (51054’48.37” O; 29²7’21.62” S), no mês de março de 2016.

Quadro 01 - Lista das espécies estudadas

\begin{tabular}{|c|l|c|}
\hline Família & \multicolumn{1}{|c|}{ Nome Científico } & Nome Popular \\
\hline Anacardiaceae & Schinus terebinthifolius Raddi & Aroeira-falsa \\
\hline Annonaceae & Annona rugulosa (Schltdl.) H.Raine & Araticum \\
\hline Boraginaceae & Cordia americana (L.) Gottschling \& J.S.Mill. & Guajuvira \\
\hline \multirow{2}{*}{ Fabaceae } & Inga vera Willd & Ingá \\
\hline \multirow{2}{*}{ Moraceae } & Ficus luschnathiana (Miq.) Miq. & Figueira \\
\cline { 2 - 3 } & Maclura tinctoria (L.) D. Don ex Steud. & Taiúva \\
\hline \multirow{2}{*}{ Myrtaceae } & Campomanesia xanthocarpa (Mart.) O. Berg & Guabiroba \\
\cline { 2 - 3 } & Myrcianthes pungens (O.Berg) D.Legrand & Guabiju \\
\hline Salicaceae & Casearia sylvestris Sw. & Chá-de-bugre \\
\hline
\end{tabular}

Fonte: Elaborado pelos autores.

As amostras foram identificadas no Laboratório do Setor de Paleobotânica e Evolução de Biomas do Museu de Ciências Naturais da Univates (SPbEvBio/MCN/UNIVATES), onde, também, foram preparadas e secas em estufa. Após a secagem, as amostras foram cortadas, sendo uma parte, devidamente identificada, depositada na coleção do SPbEvBio/ MCN/UNIVATES sem a realização do processo de carbonização e o restante carbonizado para análise da anatomia do carvão. Os lenhos não carbonizados servirão como material para comparação posterior com os fragmentos carbonizados e, também, caso haja algum problema, o processo de carbonização poderá ser refeito. Os carvóes foram preparados seguindo a metodologia descrita por Scheel-Ybert et al. (2006), para a montagem de banco de dados antracológico.

Os exemplares preparados para carbonização foram identificados com lápis no próprio lenho, envoltos em folhas de papel alumínio, identificados externamente utilizandose caneta de tinta permanente e dispostos em cadinhos contendo areia para diminuição da quantidade de oxigênio disponível, os quais foram identificados a lápis grafite. As amostras foram carbonizadas em forno mufla com temperatura de $400^{\circ} \mathrm{C}$ durante 40 minutos. 
Tanto as amostras in natura quanto as carbonizadas foram pesadas e fotografadas (FIGURA 1) para que esse parâmetro também fosse tabulado no banco de dados. Posteriormente, as amostras de carvão, seccionadas de acordo com os três planos anatômicos da madeira (transversal, longitudinal tangencial e longitudinal radial) com o auxílio de estereomicroscópio Zeiss e montadas sobre stubs para análise sob Microscópio Eletrônico de Varredura (MEV).

Figura 1 - Imagem de lenhos carbonizados e não carbonizados realizada antes da preparação para análise sob MEV. Em A: F. luschnathiana; em B, M. tinctoria.

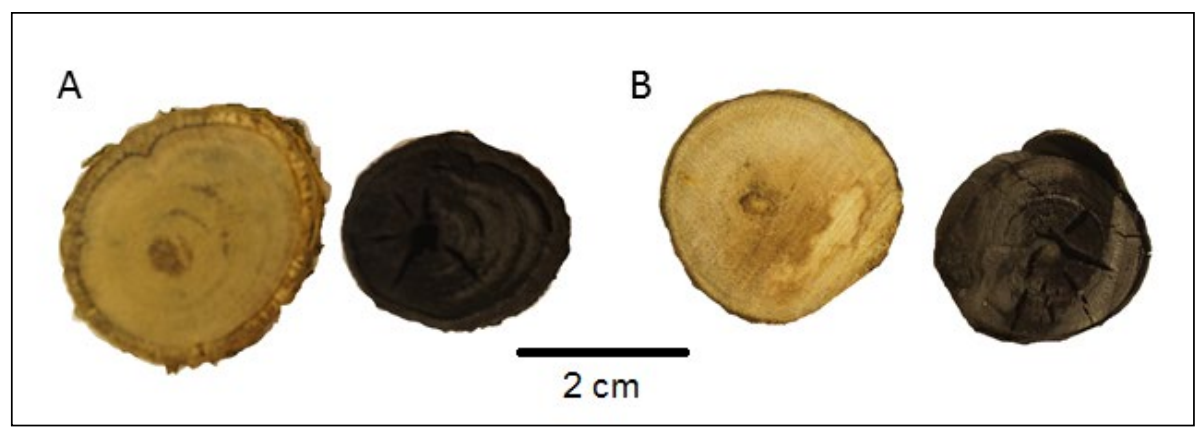

Fonte: Elaborado pelos autores.

As imagens dos fragmentos de lenhos carbonizados foram analisadas, e para cada um dos exemplares foram feitas as seguintes mensuraçóes (GONÇALVES, 2010; IAWA, 1989): diâmetro tangencial dos vasos; frequência de vasos por $\mathrm{mm}^{2}$; diâmetro tangencial das pontoaçóes intervasculares; frequência dos raios por $\mathrm{mm}^{2}$; altura dos raios; e, largura dos raios. Para cada um dos parâmetros analisados, foram efetuadas 10 (dez) mediçōes.

Os dados foram tabulados em uma planilha do Software Microsoft Office Excel 2010, onde também foram feitos os cálculos das médias e desvio padrão para cada uma das mensurações realizadas nas amostras.

\section{Resultados e Discussáo}

As características dos diferentes espécimes estudados estão resumidas nas Tabelas $1 \mathrm{e}$ 2 e ilustradas nas Figuras 2 a 4 . 
Tabela 1 - Resumo quantitativo das características anatômicas do carvão de nove espécies da flora nativa da Mata Atlântica

\begin{tabular}{l|l|l|l|l|l|l}
\hline Nome Científico & $\begin{array}{c}\boldsymbol{\emptyset} \\
\text { tangencial } \\
\mathbf{d o s} \mathbf{v a s o s} \\
(\boldsymbol{\mu m})\end{array}$ & $\begin{array}{c}\text { Frequência } \\
\mathbf{d o s} \mathbf{v a s o s} / \\
\mathbf{m m}^{\mathbf{2}}\end{array}$ & $\begin{array}{c}\boldsymbol{\emptyset} \\
\text { tangencial } \\
\mathbf{p o n t o a c ̧ o ́ e s} \\
(\boldsymbol{\mu m})\end{array}$ & $\begin{array}{c}\text { Frequência } \\
\mathbf{d o s} \mathbf{r a i o s} / \\
(\mathbf{m m})\end{array}$ & $\begin{array}{c}\text { Altura } \\
\text { raios } \\
(\boldsymbol{\mu m})\end{array}$ & $\begin{array}{c}\text { Largura } \\
\mathbf{r a i o s} \\
(\boldsymbol{\mu m})\end{array}$ \\
\hline Annona rugulosa & $\begin{array}{l}27,69 \\
(3,89)\end{array}$ & $21(2,31)$ & $3,29(0,69)$ & $4(0,74)$ & $\begin{array}{l}437,96 \\
(121)\end{array}$ & $47,44(9,29)$ \\
\hline $\begin{array}{l}\text { Campomanesia } \\
\text { xanthocarpa }\end{array}$ & $\begin{array}{l}47,20 \\
(4,83)\end{array}$ & $5(1,07)$ & $2,15(0,42)$ & $11(1,67)$ & $\begin{array}{l}172,53 \\
(46,25)\end{array}$ & $21,51(1,27)$ \\
\hline Casearia sylvestris & $\begin{array}{l}33,14 \\
(5,02)\end{array}$ & $108(2,13)$ & $1,75(0,47)$ & $7(1,46)$ & $\begin{array}{l}337,08 \\
(58,32)\end{array}$ & $32,38(6,52)$ \\
\hline Maclura tinctoria. & $\begin{array}{l}60,52 \\
(6,21)\end{array}$ & $13(1,6)$ & $6,90(1,66)$ & $12(1,45)$ & $\begin{array}{l}97,89 \\
(12,09)\end{array}$ & $14,28(2,35)$ \\
\hline Myrciantes pungens & $\begin{array}{l}35,36 \\
(3,81)\end{array}$ & $486(32,22)$ & $1,66(0,27)$ & $18(1,73)$ & $\begin{array}{l}153,28 \\
(28,33)\end{array}$ & $16,23(2,19)$ \\
\hline Ficus luschnathiana & $\begin{array}{l}39,51 \\
(5,01)\end{array}$ & $20(1,39)$ & $7,93(1,45)$ & $8(1,59)$ & $\begin{array}{l}368,57 \\
(75,10)\end{array}$ & $25,78(4,13)$ \\
\hline Inga vera & $\begin{array}{l}62,59 \\
(16,43)\end{array}$ & $35(8,61)$ & $1,31(0,36)$ & $9(1,19)$ & $\begin{array}{l}206,87 \\
(47,50)\end{array}$ & $16,57(2,51)$ \\
\hline Cordia americana & $\begin{array}{l}24,72 \\
(2,48)\end{array}$ & $58(3,33)$ & $\begin{array}{l}17,49 \\
(2,07)\end{array}$ & $6(0,71)$ & $\begin{array}{l}177,25 \\
(50,58)\end{array}$ & $35,34(7,54)$ \\
\hline Schinus teribinthifolius & $\begin{array}{l}69,45 \\
(11,01)\end{array}$ & $180(9,07)$ & $1,90(0,57)$ & $10(1,12)$ & $\begin{array}{l}199,02 \\
(47,82)\end{array}$ & $22,45(1,86)$ \\
\hline
\end{tabular}

Fonte: Elaborado pelos autores

Tabela 2 - Resumo qualitativo das características anatômicas do carvão de nove espécies da flora nativa da Mata Atlântica

\begin{tabular}{l|l|l|l|l|l|l|l|l}
\hline \multicolumn{1}{c|}{$\begin{array}{c}\text { Nome } \\
\text { Científico }\end{array}$} & AC & \multicolumn{1}{|c|}{ PP } & \multicolumn{1}{|c|}{ PI } & \multicolumn{1}{|c|}{ PA } & \multicolumn{1}{c|}{ LR } & CCR & EE & IM \\
\hline $\begin{array}{l}\text { Annona } \\
\text { rugulosa }\end{array}$ & $\mathrm{X}$ & Simples & Alternas & Aliforme & $\begin{array}{l}1-3, \\
\text { (eventual) }\end{array}$ & A & Irregular & X \\
\hline $\begin{array}{l}\text { Campomanesia } \\
\text { xanthocarpa }\end{array}$ & $\checkmark$ & Simples & Alternas & $\begin{array}{l}\text { Confluente } \\
\text { unilateral }\end{array}$ & $1-3$ & $\mathrm{~B}$ & Irregular & $\mathrm{X}$ \\
\hline $\begin{array}{l}\text { Casearia } \\
\text { sylvestris }\end{array}$ & $\mathrm{X}$ & Escalariformes & Alternas & Difuso & $1-3$ & $\mathrm{D}$ & $\mathrm{X}$ & $\mathrm{X}$ \\
\hline $\begin{array}{l}\text { Maclura } \\
\text { tinctoria. }\end{array}$ & $\mathrm{X}$ & Simples & Alternas & Confluente & $1-3$ & $\mathrm{~A}$ & $\mathrm{X}$ & $\mathrm{X}$ \\
\hline $\begin{array}{l}\text { Myrciantes } \\
\text { pungens }\end{array}$ & $\checkmark$ & Simples & Alternas & Em faixas & $1-3$ & $\mathrm{D}$ & $\mathrm{X}$ & $\mathrm{X}$ \\
\hline $\begin{array}{l}\text { Ficus } \\
\text { luschnathiana }\end{array}$ & $\checkmark$ & Simples & Alternas & $\begin{array}{l}\text { Paratraqueal- } \\
\text { unilateral }\end{array}$ & $1-3$ & $\mathrm{C}$ & $\mathrm{X}$ & $\mathrm{X}$ \\
\hline
\end{tabular}




\begin{tabular}{l|l|l|l|l|l|l|l|l}
\hline \multicolumn{1}{c|}{$\begin{array}{c}\text { Nome } \\
\text { Científico }\end{array}$} & AC & \multicolumn{1}{|c|}{ PP } & \multicolumn{1}{|c|}{ PI } & \multicolumn{1}{c|}{ PA } & LR & CCR & \multicolumn{1}{c|}{ EE } & IM \\
\hline Inga vera & $\checkmark$ & Simples & Alternas & $\begin{array}{l}\text { Paratraqueal } \\
\text { confluente }\end{array}$ & $1-3$ & A & X & X \\
\hline $\begin{array}{l}\text { Cordia } \\
\text { americana }\end{array}$ & $\checkmark$ & Simples & Alternas & $\begin{array}{l}\text { Paratraqueal } \\
\text { confluente }\end{array}$ & $1-3$ & D & Raios & X \\
\hline $\begin{array}{l}\text { Schinus } \\
\text { teribinthifolius }\end{array}$ & $\checkmark$ & Simples & Alternas & $\begin{array}{l}\text { Paratraqueal- } \\
\text { unilateral }\end{array}$ & $1-3$ & C & X & X \\
\hline
\end{tabular}

Fonte: Elaborado pelos autores.

Legenda: AC: anéis de crescimento; PP: placas de perfuração; PI: pontoaçôes intervasculares; PA: parênquima axial; LR: largura de raio; CCR: composiçáo das células de raio (A: todas as células de raio procumbentes; $\mathrm{B}$ : corpo das células de raio procumbentes com uma fileira de células marginais eretas ou quadradas; C: corpo das células de raio procumbentes com mais de 2 a 4 fileiras de células marginais eretas ou quadradas; D: células de raio procumbentes, quadradas e eretas misturadas através do raio); EE: estrutura estratificada; IM: inclusóes minerais; X: indistinto ou ausente; $\sqrt{ }$ : presente.

Figura 2 - Imagens sob microscopia eletrônica de varredura dos três planos anatômicos da madeira, transversal, longitudinal tangencial, longitudinal radial. Annona rugulosa (A-C). Ficus luschnathiana (D-F). Maclura tinctoria (G-I)
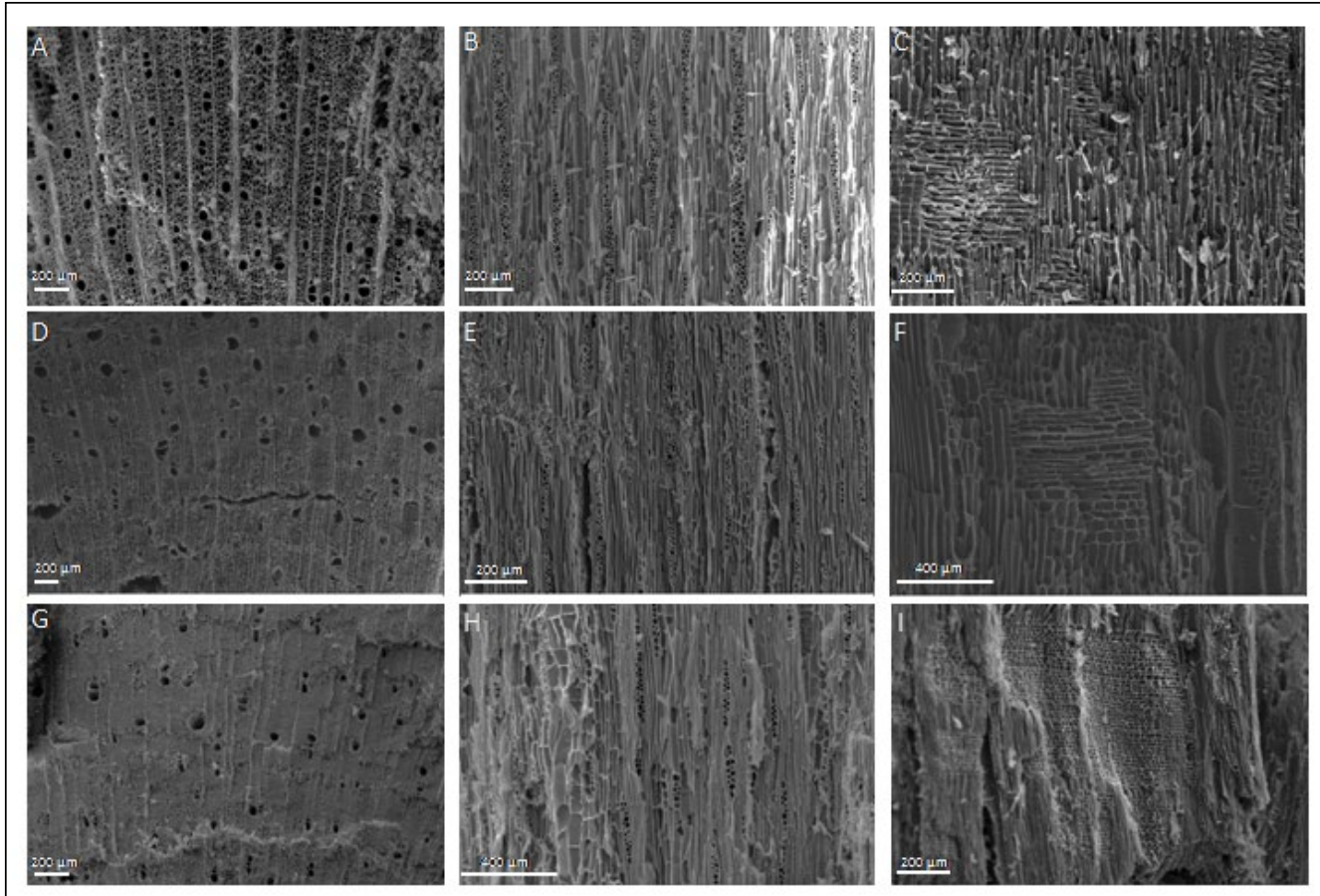

Fonte: Elaborado pelos autores.

A formação de carvão pode ocorrer tanto com o aquecimento do lenho em ambientes com quantidades de oxigênio limitadas quanto na sua ausência. No primeiro caso, o processo 
de queima ocorre, liberando substâncias voláteis que se inflamam, produzindo as chamas. Nesse processo, a combustáo prossegue até a formação das cinzas, ou, caso a combustấo seja incompleta, resulta na produção de carvão. No segundo caso, também há liberaçáo de substâncias voláteis, mas não haverá formação de chamas, resultando diretamente na formação do carvão (BRAADBART; POOLE, 2008).

Figura 3 - Imagens sob microscopia eletrônica de varredura dos três planos anatômicos da madeira, transversal, longitudinal tangencial, longitudinal radial. Campomanesia xanthocarpa (A-C). Casearia sylvestris (D-F). Schinus teribinthifolius (G-I)

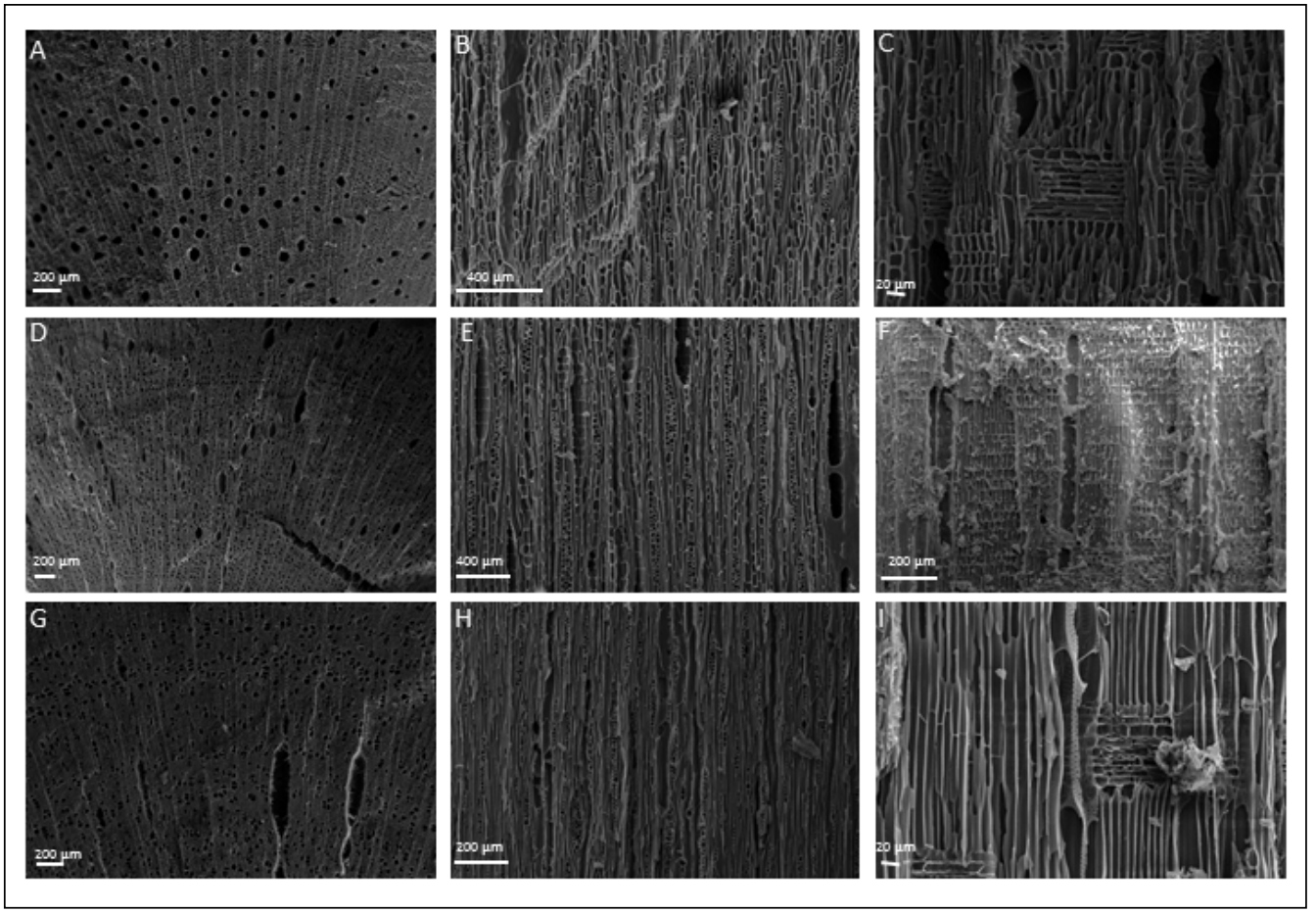

Fonte: Elaborado pelos autores. 
Figura 4 - Imagens sob microscopia eletrônica de varredura dos três planos anatômicos da madeira, transversal, longitudinal tangencial, longitudinal radial. Cordia americana (A-C). Myrciantes pungens (D-F). Inga vera (G-I)

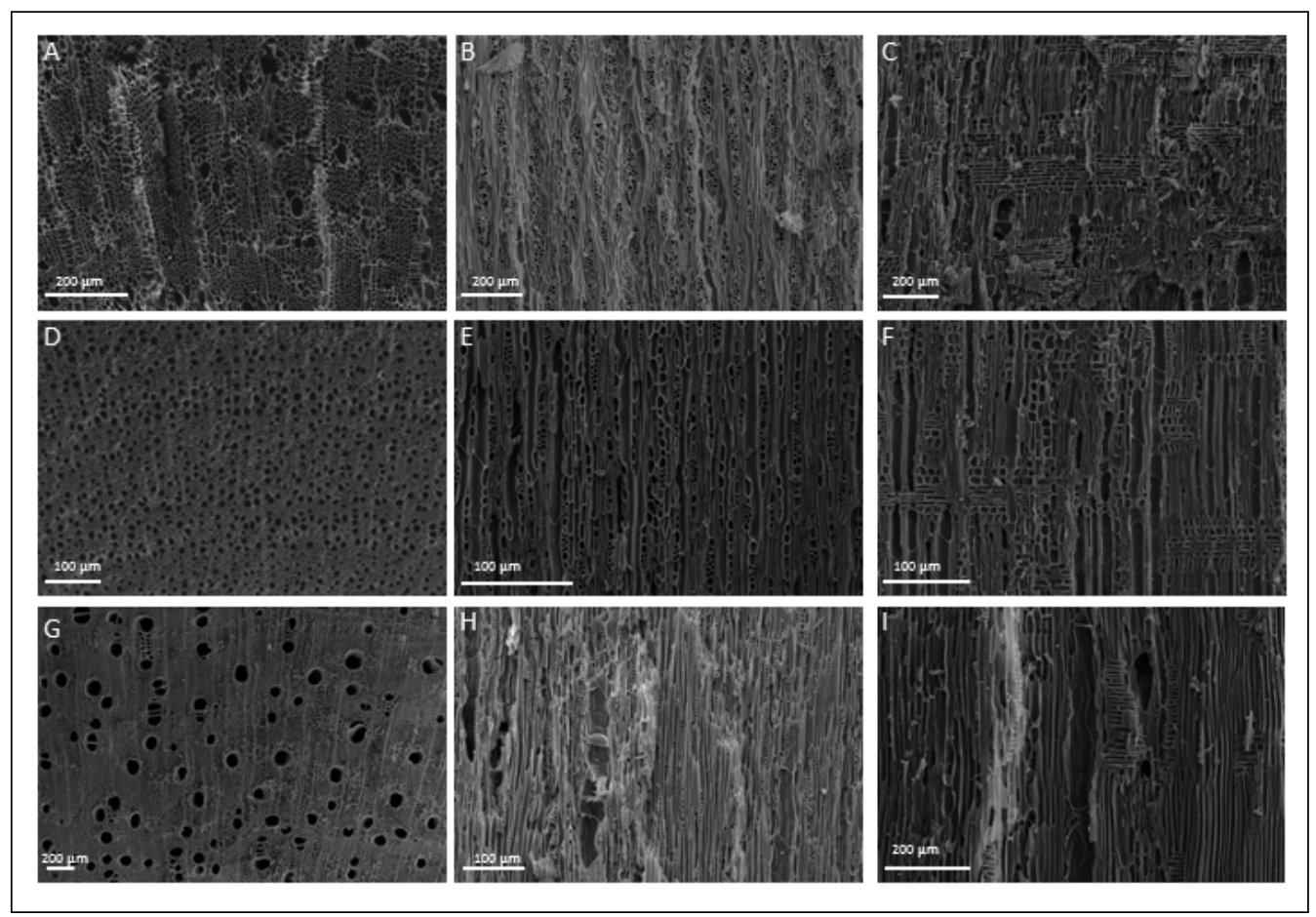

Fonte: Elaborado pelos autores.

Devido ao processo de carbonização no forno mufla, os lenhos carbonizados tiveram considerável redução de massa em virtude da perda de água. O peso das amostras, pósqueima, foi reduzido, em média, em 75,67\% da massa total da amostra in natura (TABELA 03 ), sendo que $S$. teribinthifolius teve maior redução de massa, $82,30 \%$. Já a espécie que teve menor redução no peso pós-queima em relação às amostras não queimadas foi $C$. americana $(67,55 \%)$.

Em estudos realizados por McGinnes et al. (1971) com a espécie Quercus alba L. a perda de massa foi entre 34,5 a $41,5 \%$, em temperaturas entre $270-400^{\circ} \mathrm{C}$, além da amostra carbonizada apresentar alteração nas paredes celulares, resultanto na homogeneização destas com aparência amorfa. Prior e Alvin (1983), em estudos realizados com Dichrostachys cinerea e Salix subserrata, descobriram que acima de $300^{\circ} \mathrm{C}$ as paredes celulares começam a adquirir característica amorfa.

Kim e Hanna (2006) em estudo realizado com Q. variabilis BL, perceberam perda de $74,4 \%$ na amostra de carvão após carbonização a $400^{\circ} \mathrm{C}$. Segundo o estudo dos autores, as características originais foram mantidas, não havendo alteração nas paredes celulares. Já Kwon et al. (2009), em estudo sobre a mesma espécie, inferiram que acima de $350^{\circ} \mathrm{C}$ não é possível verificar a diferenciação da lamela média. 
A não alteração na estrutura das paredes celulares observadas por Kim e Hanna (2006) difere dos resultados encontrados no estudo de McGinnes et al. (1971) o qual utilizou o mesmo gênero, bem como de Prior e Alvin (1983) e Kwon et al. (2009), no que se refere a modificaçóes na estrutura da parede celular.

$\mathrm{O}$ presente estudo corrobora com inferências dos autores acima, visto que nas espécies analisadas ocorreu homogeneização das paredes celulares em todas as amostras, em uma temperatura de $400^{\circ} \mathrm{C}$ pelo período de 40 minutos.

Tabela 03 - Massa das amostras de carvão, lenho in natura, suas respectivas médias e percentual de redução de massa em relação ao peso dos lenhos in natura e os carbonizados (charcoals)

\begin{tabular}{|c|c|c|c|c|c|c|c|}
\hline $\begin{array}{c}\text { Nome } \\
\text { Científico }\end{array}$ & $\begin{array}{c}\text { Charcoal } \\
1(\mathrm{~g})\end{array}$ & $\begin{array}{c}\text { Charcoal } \\
2(\mathrm{~g})\end{array}$ & $\begin{array}{c}\text { Lenho in } \\
\text { natura } 1 \\
(\mathrm{~g})\end{array}$ & $\begin{array}{c}\text { Lenho in } \\
\text { natura } 2 \\
(\mathrm{~g})\end{array}$ & $\begin{array}{c}\text { Média } \\
\text { charcoals } \\
\text { (g) }\end{array}$ & $\begin{array}{c}\text { Média } \\
\text { lenhos } \\
(\mathrm{g})\end{array}$ & $\begin{array}{c}\text { Percentual de } \\
\text { reduçáo de } \\
\text { massa }(\%)\end{array}$ \\
\hline $\begin{array}{l}\text { Annona } \\
\text { rugulosa }\end{array}$ & 0,9562 & 0,9094 & 3,4941 & 3,1414 & $\begin{array}{l}0,9328 \\
(0,033)\end{array}$ & $\begin{array}{l}3,3178 \\
(0,249) \\
\end{array}$ & 71,88 \\
\hline $\begin{array}{l}\text { Campomanesia } \\
\text { xanthocarpa }\end{array}$ & 1,2283 & 1,0645 & 4,2393 & 4,0219 & $\begin{array}{l}1,1464 \\
(0,115)\end{array}$ & $\begin{array}{l}4,1306 \\
(0,153)\end{array}$ & 72,24 \\
\hline $\begin{array}{l}\text { Casearia } \\
\text { sylvestris }\end{array}$ & 1,5048 & 1,4296 & 7,3795 & 7,5851 & $\begin{array}{l}1,4672 \\
(0,053)\end{array}$ & $\begin{array}{r}7,4823 \\
(0,145) \\
\end{array}$ & 80,39 \\
\hline $\begin{array}{l}\text { Maclura } \\
\text { tinctoria. }\end{array}$ & 1,2616 & 1,363 & 6,3683 & 5,5939 & $\begin{array}{l}1,3123 \\
(0,071) \\
\end{array}$ & $\begin{array}{l}5,9811 \\
(0,547) \\
\end{array}$ & 78,06 \\
\hline $\begin{array}{l}\text { Myrciantes } \\
\text { pungens }\end{array}$ & 1,4973 & 1,9007 & 6,9895 & 6,0393 & $\begin{array}{l}1,699 \\
(0,285)\end{array}$ & $\begin{array}{l}6,5144 \\
(0,671) \\
\end{array}$ & 73,91 \\
\hline $\begin{array}{l}\text { Ficus } \\
\text { luschnathiana }\end{array}$ & 1,4068 & 1,3468 & 7,7228 & 7,1317 & $\begin{array}{l}1,3768 \\
(0,042) \\
\end{array}$ & $\begin{array}{l}7,4273 \\
(0,418) \\
\end{array}$ & 81,46 \\
\hline Inga vera & 0,6716 & 1,0486 & 2,863 & 2,7207 & $\begin{array}{l}0,8601 \\
(0,266) \\
\end{array}$ & $\begin{array}{l}2,7919 \\
(0,100) \\
\end{array}$ & 69,19 \\
\hline $\begin{array}{l}\text { Cordia } \\
\text { americana }\end{array}$ & 0,6864 & 0,7968 & 2,9609 & 1,6108 & $\begin{array}{l}0,7416 \\
(0,078) \\
\end{array}$ & $\begin{array}{l}2,2859 \\
(0,954) \\
\end{array}$ & 67,55 \\
\hline $\begin{array}{l}\text { Schinus } \\
\text { teribinthifolius }\end{array}$ & 0,7910 & 0,7818 & 4,4585 & 4,4310 & $\begin{array}{l}0,7864 \\
(0,006) \\
\end{array}$ & $\begin{array}{l}4,4448 \\
(0,019) \\
\end{array}$ & 82,30 \\
\hline
\end{tabular}

Fonte: Elaborado pelos autores

Afonso et al. (2015), analisaram espécies nativas de Moçambique e também realizaram carbonização das amostras. Das nove espécies analisadas pelos autores, sete apresentaram algum tipo de ruptura. Também foram observadas rupturas em várias amostras, como foi o caso de F. luschnathiana (FIGURA 5), C. sylvestris (fissuras paralelas aos raios), M. tinctoria, C. americana (fissuras paralelas e perpendiculares aos raios), S. teribinthifolius, M. pungens (fissura perpendicular aos raios). Nas demais amostras fissuras não foram observadas. A ocorrência de rupturas nas amostras de carvão vegetal já era esperada visto que após a carbonização ocorre a contração do material podendo ou não ocorrer fissuras, mas a sua ocorrência não compromete a identificação dos fragmentos de carvão estudados. 
Figura 5 - Fissura paralela aos raios em $F$ luschnathiana

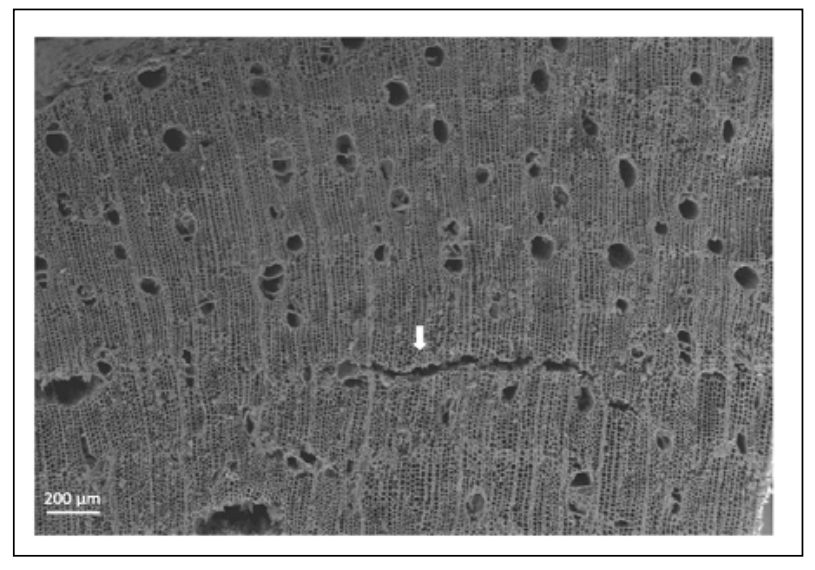

Fonte: Elaborado pelos autores

Outros estudos sobre anatomia de carvão, e citados por Afonso et al. (2015), também observaram a ocorrência de fissuras, como é o caso de Corymbia citriodora (Hook.) K.D.Hill et L.A.S. Johnson, C. maculata (Hook.) K.D. Hill et L.A.S. Johnson, Eucalyptus dunnii Maiden, E. tereticornis Sm. e E. viminalis La-bill. (GONÇALVES et al., 2014), Quercus variabilis, Q. robur L. (KIM; HANNA, 2006) e P. alba L. (MCGINNES et al., 1971), cujas rupturas deram-se nos raios; E. dunnii (GONÇALVES et al., 2014), no parênquima axial; e, Mimosa tenuiflora (Willd.) Poiret e M. ophthalmocentra Martius (DIAS LEME et al., 2010), nas fibras.

De acordo com Afonso et al. (2015), as alteraçóes anatômicas geradas devido à carbonização de lenhos não influenciam na distinção de espécies. Dessa forma, estudos sobre anatomia de carvão podem ser utilizados, além de estudos arqueobotânicos, paleobotânicos e botânicos, como uma ferramenta fiscalizatória, contribuindo para coibir o uso de espécies nativas, não licenciadas pelos órgãos competentes (FEPAM, IBAMA, órgáos licenciadores municipais), como fonte energética. Além disso, as inferências acerca dos paleoambientes regionais permitirão a definição de açôes relacionadas ao planejamento e gestão ambiental regional, quando estas estiverem relacionadas à recuperaçấo da cobertura vegetal.

\section{Conclusóes}

O conhecimento da anatomia do carvão vegetal pode auxiliar na identificação de fragmentos carbonizados encontrados em ambientes pretéritos, visto que o fogo não destrói as estruturas celulares e o carvão se preserva no sedimento. Dessa forma é possível realizar comparaçóes de carvões atuais com fragmentos vegetais do passado a fim de fazer reconstruçôes paleoambientais e paleoetnológicas.

Diante disso, é importante que se façam coleçôes de referência ou banco de dados antracológicos com o maior número possível de espécies para facilitar o processo de comparação e por consequência de reconstruçóes paleoambientais. Tendo em mãos as 
características pretéritas dos ambientes regionais, elas servirão de base para o desenvolvimento de açôes que visem a recuperação da vegetação nativa do Vale do Taquari.

Todavia, mais estudos acerca da anatomia de carvão devem ser realizados para que um banco de dados antracológico expressivo possa ser constituído, o que necessita de tempo e recursos.

Além disso, o conhecimento das características anatômicas dos componentes da flora reginal atual pode ser utilizado como ferramenta na realização de ações de fiscalização, visto que as espécies aqui estudadas são exemplares da vegetação nativa, cujo corte para fins energéticos é vedado sem o devido licenciamento. A disseminação de estudos anatômicos de carvão é importante para que se possa ter um maior controle da produção deste tipo de fonte energética, coibindo as ações danosas ao ambiente e contribuindo para a conservação das espécies nativas do Brasil.

Considerando que os resultados aqui apresentados se referem a nove espécies da flora da regiấo do Vale do Taquari, é fundamental que as coletas e análises anatômicas envolvendo outros táxons tenham continuidade, possibilitando a constituição de uma base de dados representativa. Neste sentido, é mandatório que novos trabalhos sobre a temática sejam levados a cabo para que uma maior eficiência nos estudos comparativos seja atingida.

\section{Agradecimentos}

Os autores agradecem a Capes pelas bolsas de pós-graduação e Univates, CNPq, CAPES e FAPERGS pelo suporte financeiro. A. Jasper agradece ao CNPq pela Bolsa de Produtividade em Pesquisa. Os autores agradecem, ainda, as valiosas contribuiçóes dadas pelos Revisores Anônimos A e B e pelo Prof. Dr. Alexandre André Feil, Editor Geral da Estudo \& Debate.

\section{Referências}

AFONSO, C. M. I.; GONÇALVES, T. A. P.; MUÑIZ, G. I. B.de; MATOS, J. L. M.de; NISGOSKI, S. Mozambique's charcoals: anatomy of nine native species. Bosque v.36, n. 1: p. 105-112. 2015.

ARCHAEOBOTANY. Dictionary.com. Collins English Dictionary - Complete \& Unabridged 10th Edition. Harper Collins Publishers. Disponível em http://dictionary. reference.com/browse/archaeobotany. Acesso em: 01 de fevereiro de 2016.

BEUREN, J.; SECCHI, M.I.; JASPER, A.; WOLF, S.; MACHADO, N. T. G. Análise de carvão vegetal macroscópico em porções do Sítio Arqueológico RS-T-101, Marques de Souza/Rio Grande do Sul, Brasil. Caderno de Pesquisa - Serie Biologia. Santa Cruz do Sul. v. 24, n. 3, p. 24-37. 2012.

BLACK, M.; BEWLEY, J. D.; HALMER, P. The Encyclopedia of Seeds: Science, Technology and Uses. Wallengford, UK. CABI. 2006. 
BRAADBAART, F; POOLE, I. Morphological, chemical and physical changes during charcoalification of wood and its relevance to archaeological contexts. Journal of Archaeological Science, New York, v. 35, p. 2434-2445. 2008. http://dx.doi. org/10.1016/j.jas.2008.03.016

DIAS LEME C.L.; CARTWRIGHT, C.; GASSON, P. Anatomical changes to the wood of Mimosa ophthalmocentra and Mimosa tenuiflora when charred at different temperatures. IAWA Journal. v. 31, n. 3, p. 333-351. 2010. DOI: 10.1163/22941932-90000027

GONÇALVES, T. A. P. Anatomia do lenho e do carvão de espécies arbóreas do cerrado no estado de Sáo Paulo, Brasil. Dissertação de Mestrado. Faculdade de Ciências Agronômicas da UNESP. 113p. 2010.

GONÇALVES, T. A. P.; MARCATI, C.R.; SCHEELYBERT, R. The effect of carbonization on wood structure of Dalbergia violacea, Stryphnodendron polyphyllum, Tapirira guianensis, Vochysia tucanorum, and Pouteria torta from the Brazilian cerrado. IAWA Journal, v. 33, p. 73-90. 2012.

GONÇALVES T.A.P; BALLARIN, A.W.; NISGOSKI, S.; MUÑIZ, G.I.B. A contribution to the identification of charcoal origin in Brazil i: anatomical characterization of Corymbia and Eucalyptus. Maderas Ciencia y Tecnologia, v. 16, n. 3, p. 323-336. ISSN 0718-221X. 2014.

HADLER, P.; DIAS, A. S.; BAUERMANN, S. G. Multidisciplinary studies of Southern Brazil Holocene: Archaeological, palynological and paleontological data. Quaternary Intenational. 2012.: <doi: 10.1016/j.quaint.2012.09.026>.

IAWA Committee. IAWA List of microscopic features for hardwood identification. IAWA Bulletin. v. 10, n. 3, p. 219-332. 1989.

JONES, T. P.; CHALONER, W. Charcoal, its recognition and palaeoatmospheric significance. Palaeogeography, Palaeoclimatology, Palaeoecology, v. 97, n. 1-2, p. 3950. Dez. 1991.

KIM, N.H.; HANNA, R.B. Morphological characteristics of Quercus variabilis charcoal prepared at different temperatures. Wood Science and Technology, v. 40, p. 392-401. 2006.

KWON, S. M., N-H KIM, D. S. CHA. An investigation on the transition characteristics of the wood cell walls during carbonization. Wood Science and Technology, v. 43, n. 5-6, p. 487-498. 2009.

LEONHARDT, A. Mudanças vegetacionais e climáticas no Planalto leste do Rio Grande do Sul, Brasil, durante os últimos 25000 anos. Dissertação de Mestrado, 
Programa de Pós-Gradudação em Botânica, Universidade Federal do Rio Grande do Sul, Porto Alegre, 124 p. 2007.

MARGUERIE, D; HUNOT, J-Y. Charcoal analysis and dendrology: data from archaeological sites in north-western France. Journal of Archaeological Science, v. 34, p. 1417 - 1433. 2007.

MATEUS, J. A. Arqueologia da Paisagem e Paleoecologia. Almadán, II série, no 5 "Especial Ciência”. Outubro de 1996.

MCGINNES, E. A.; KANDEEL, S. A.; SZOPA, P. S. Some structural changes observed in the transformation of wood into charcoal. Wood and Fiber, Monona, v. 3, n. 2, p. 77 83. 1971.

MUNIIZ, G.I.B.; CARNEIRO, M.E.; NISGOSKI, S.; RAMIREZ, M.G.L.; MAGALHÃES, W.L.E. SEM and NIR characterization of four charcoal species. Wood Science and Technology, v. 47, n. 4, p. 815-823. 2013.

NISGOSKI, S; MAGALHÁES, W. L. E.; BATISTA, F. R. R.; FRANÇA, R. F; MUNIZ, G. I. BOLZON DE. Anatomical and energy characteristics of charcoal made from five species. Acta Amaz., Manaus, v. 44, n. 3, p. 367-372. Set. 2014 . Doi: $10.1590 / 1809-4392201304572$

PRIOR, J., ALVIN, K. L. Structural changes on charring woods of Dichristachys and Salix from Southern Africa. IAWA Bulletin v. 4, n. 4, p. 197-206. 1983.

PRIOR, J.; GASSON, P. Anatomical changes on six African hardwoods. IAWA Journal, v. 14 , p. $77-86.1993$.

SCHEEL-YBERT, R. CAROMANO, C. F.; CASCON, L. M.; BIANCHINI, G. F.; BEAUCLAIR, M. Estudos de paleoetnobotânica, paleoambiente e paisagem na Amazônia Central e o exemplo do sudeste-sul do Brasil. In: Pereira, E.; Guapindaia, V. (eds.)

Arqueologia Amazônica. Belém: Museu Paraense Emílio Goeldi. v. 2, p. 909-935. 2010.

SCHEEL, R.; GASPAR, M.D.; YBERT, J.P. A ANATOMIA DOS CARVÓES PRÉHISTÓRICOS. ARQUEOLOGIA ENCONTRA RESPOSTAS EM RESTOS DE FOGUEIRAS E INCÊNDIOS FLORESTAIS. Ciência Hoje, v. 21, n. 122, p. 66-69. 1996.

SCHEEL-YBERT, R.; GOUVEIA, S.E. M.; PESSENDA, L.C.R.; COUTINHO, L. M.; BOULET, R. HOLOCENE PALAEOENVIRONMENTAL EVOLUTION OF THE CERRADO AND SEMIDECIDUOUS FOREST ZONE IN THE SÃO PAULO STATE (BRAZIL), BASED ON ANTHRACOLOGY AND SOIL D13C ANALYSIS. The Holocene, v. 13, n.1, p. 73-81. 2003. 
SCHEEL-YBERT, R. KLÖKLER, D.; GASPAR, M.D.; FIGUTI, L. Proposta de amostragem padronizada para macrovestígios bioarqueológicos: antracologia, arqueobotânica, zooarqueologia. Revista do Museu de Arqueologia e Etnologia, v. 1516, p. 139-163. 2006.

SCHEEL-YBERT, R. ANTRACOLOGIA: MÉTODOS E PERSPECTIVAS. Disponível on line in: "Arqueologia Brasileira": Arqueologia em Conexão no 6. 2000.

SCHEEL-YBERT, R. Antracologia: preservados pelo fogo. In: M.D. Gaspar; S.M. Mendonça de Souza. (Org.). Protocolos para pesquisas de campo em sambaquis. 1ed. Erechim: Habilis, p. 193-218. 2013.

SCHEEL-YBERT, R. Teoria e métodos em antracologia. 1. Consideraçóes teóricas e perspectivas. Arquivos do Museu Nacional, v. 62, n. 1, p. 3-14. 2004 b.

SCHEEL-YBERT, R. Teoria e métodos em antracologia. 2. Técnicas de campo e de laboratório. Arquivos do Museu Nacional, v. 62, n. 4, p. 343-356. 2004 a.

SCHMIDT, E. O. Avaliação antracológica de fragmentos de carvão vegetal fóssil em porçóes do sítio arqueológico RS T 114: um estudo multidisciplinar para a determinação de histórico ambiental. Dissertação de Mestrado (Programa de PósGraduação em Ambiente e Desenvolvimento - Centro Universitário UNIVATES). 2010.

SCOTT A. C.; DAMBLON F. Charcoal: Taphonomy and significance in geology, botany and archaeology. Palaeogeography, Palaeoclimatology, Palaeoecology, v. 291, p.1-10. 2010 .

SCOTT, A. C. The pre-quaternary history of fire.Palaeogeography, Palaeoclimatology, Palaeoecololgy, v. 164, p. 281-329. 2000.

SCOTT, A. C. Charcoal recognition, taphonomy and uses in palaeoenvironmental analysis. Palaeogeography, Palaeoclimatology, Palaeoecology, v. 291, p. 11-39. 2010.

SECCHI, M.I. Avaliação antracológica em sítios pré-coloniais como ferramenta para a análise da História Ambiental da Bacia Hidrográfica do Rio Forqueta, Rio Grande do Sul, Brasil. Dissertação de Mestrado (Programa de Pós-Graduação em Ambiente e Desenvolvimento - Centro Universitário UNIVATES), Brasil. 83p. 2012.

TERESO, J. P. V. Introduçáo ao estudo de macro-restos vegetais em sítios arqueológicos. $1^{\text {a }}$ Ed. Faculdade de Ciências do Porto. Maio 2008.

TERESO, J. P. V. Paleoetnobotânica do povoado romano da Terronha de Pinhovelo (NE transmontano). Dissertação de Mestrado, Faculdade de Ciências. Universidade do Porto. 2007. 